\title{
Ganglioside research: New trends and reflections
}

\author{
Yoshitaka Nagai \\ Mitsubishi Kasei Institute of Life Sciences* and Glycobiology Research Group, Frontier \\ Research Program, The Institute of Physical and Chemical Research (RIKEN) \\ *11, Minamiooya, Machida-city, Tokyo 194, Japan.
}

\begin{abstract}
Glycobiology of gangliosides (a family of sialic acid-containing glycosphingolipids) is rapidly expanding for the past five years, focusing on the functional and molecular biological aspects. Two contrasted trends arise in the studies of bioactive gangliosides in terms of biosignaling mechanism. Many glycosyltransferases were now cloned and progress in molecular genetic approaches using these clones present new enigmas concerning glycolipid functions.
\end{abstract}

Gangliosides, a family of sialic acid-containing glycosphingolipids, were first discovered by E. Klenk in 1935 in the brain of a patient with an inherited metabolic disorder (infantile amaurotic idiocy) called TaySachs disease. The disease is known to be one of the lysosomal diseases, which is deficient in a catabolic enzyme of gangliosides, $\beta$-1,4-N-acetylgalactosaminidase, resulting in the accumulation of GM2 gangliosides (Fig. 1 and 2). Tay-Sachs ganglioside GM2 opened a path to the studies of gangliosides as well as sialic acids and related compounds. Brain tissues are uniquely rich in a particular family of gangliosides different from those in extraneural tissues. The brain gangliosides are characterized principally by having $\mathrm{N}$-acetylgalactosamine as a constituent, while the latter by $\mathrm{N}$-acetylglucosamine. The first extraneural ganglioside, GM3, was isolated from equine erythrocytes by T. Yamakawa in 1951. Up to the present, the number of gangliosides so far determined in vertebrates is around 126 and the number of brain gangliosides is presumed to be more than forty, most of which represent minor components (Fig. 3) (ref. 1). As seen in other glycosphingolipids extraneural gangliosides of vertebrates show distinct variation in molecular species according to animal species, organ, tissue and cell types. In other words, they may constitute cell surface markers or the face of cells. In contrast, molecular species of brain gangliosides are relatively conserved.

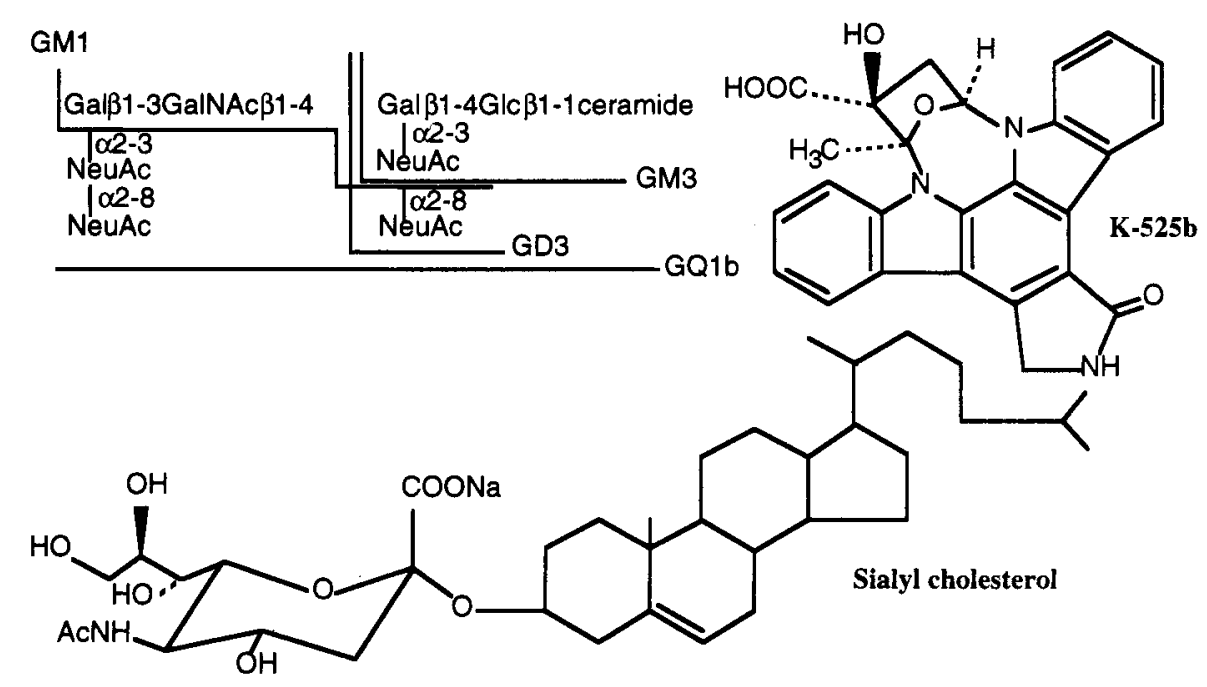

Fig. 1 


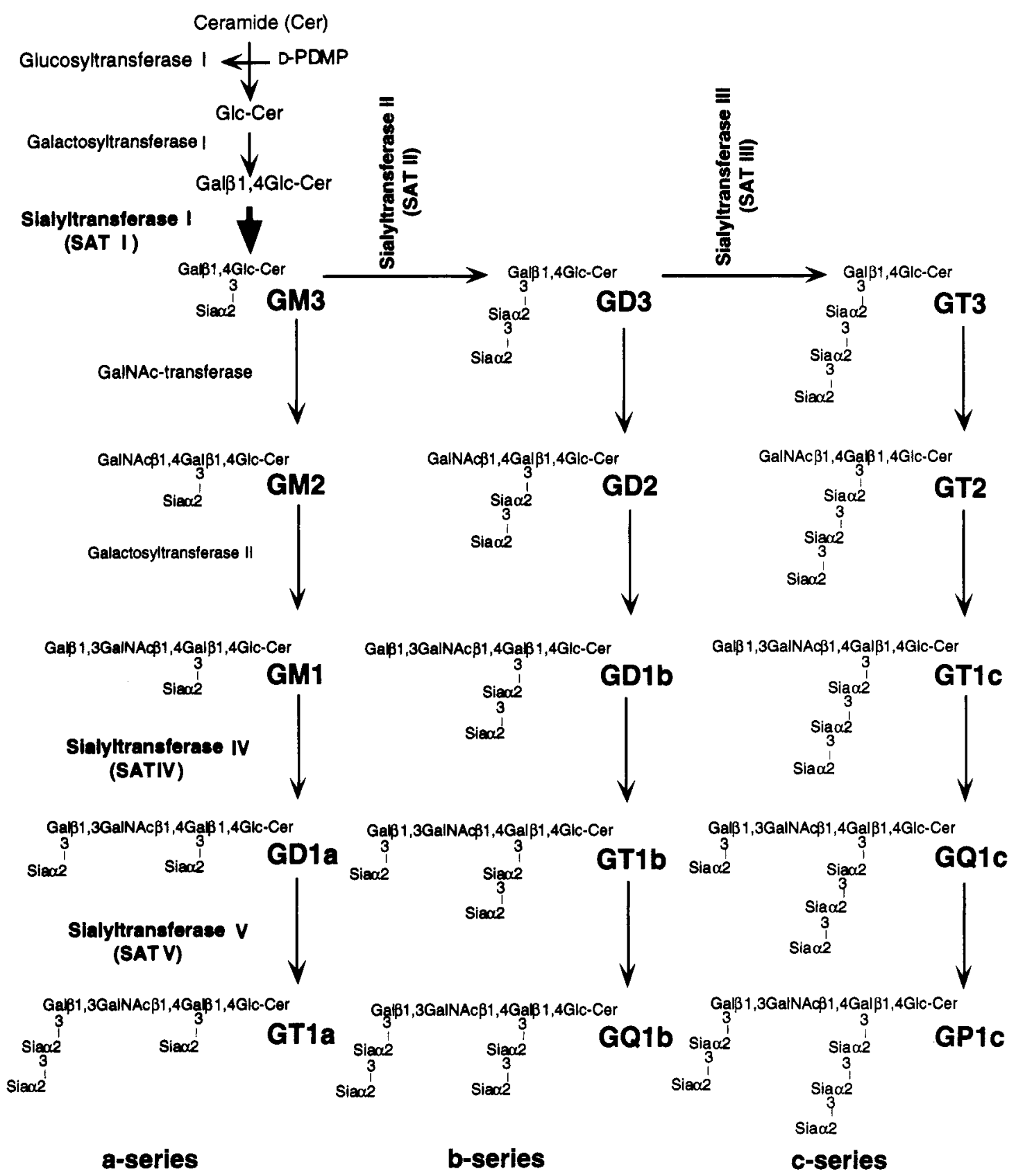

Fig. 2 Biosynthetic Pathway for Gangliosides

\section{CELL-CELL RECOGNITION AND ADHESION}

The expression of the carbohydrate face is developmentally regulated in a stage-specific manner. It is likely that nature may utilize the face as means of cell-cell recognition, adhesion, cell migration guidance in organogenesis, morphogenesis and related biological phenomena. Cell adhesion molecules or their counterpart ligands frequently are glycoconjugates. For example, E-selection on endothelial cells of blood vessels and sialyl $\mathrm{Le}^{\mathrm{x}}$ of leukocyte surface as ligand play an essential role in initial rolling step at the inflammatory injured tissue region of the leukocytes moving along the wall of blood capillary. Importance of such carbohydrate-mediated interactions between adhesion molecules and ligands are also recognized in angiogenesis, tumor metastasis, microbial infection, specific cell binding of bacterial toxins 
and sometimes xenotransplantation. The first international symposium on "anti-adhesion therapy" focusing on microbial diseases was held in Israel in 1996. The lipidized carbohydrate chains (neoglycolipids) are expected to be hopeful candidates for the strategy of anti-adhesion therapy. Moreover, carbohydrate replica peptides or mimics recently developed by phage display methodology using random peptide epitope library are found to be useful for that purpose, for example, prevention of tumor metastasis (ref. 2).

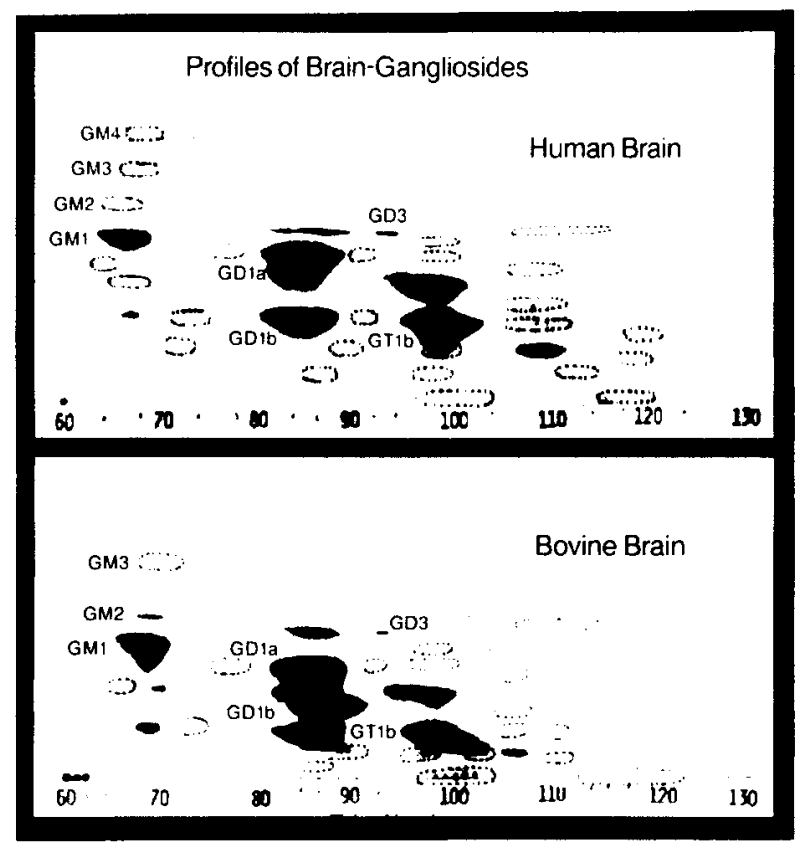

Fig. 3 Profiles of Brain-Gangliosides

\section{BRAIN AND SUGAR CHAINS}

Brain is the most intricate organ that highly develops the mechanism of cell-cell interactions accompanying adhesion and recognition as typically recognized in the formation and plasticity of neuron network and also in neuron-glia structural and functional collaboration. It is very likely that glycoconjugates play essential roles also in this research area. It is interesting to note that the biosynthesis of a sugar chain is catalyzed step by step by specific enzymes (glycosyltransferases) of which activity and specificity are under the influence of several environmental parameters (ions, salts, temperature, concentration and competition of sugar donors and acceptors, etc.), giving a certain fuzzy nature to the synthetic mechanism. This is sharply contrasted with the template-dependent mechanism of the synthesis of nucleic acids and proteins, that is, rigorously controlled mechanism. A great structural diversity of the sugar chain and the fuzzy character of its cellular expression may fulfill a condition for the plasticity of brain. At the present, however, we have little knowledge about the sugar chains of brain tissues except for glycosphingolipids, in particular, gangliosides. Brain gangliosides are uniquely distributed in different regions of brain tissues and characteristically change in the course of embryonic and postnatal development. But what are their functional roles?

\section{TWO TRENDS IN STUDIES OF BIOACTIVE GANGLIOSIDES}

Carbohydrate recognition in cellular functions does not always remain mere binding of either ligand to cell or one cell to another, but frequently triggers subsequent cellular response which is mediated by biosignaling mechanism. Up to the present, there seem to be two different trends in the research of bioactive gangliosides. Bremer, E. and Hakomori, S.-I. in 1982 (ref. 3) reported that ganglioside GM3 and not GM1 added to cultured hamster fibroblast cells may regulate the function of the receptor for fibroblastic growth factor (FGF). Then, we reported in 1983 that tetrasialoganglioside GQ1b as a minor 
brain ganglioside when added to cultured human neuroblastoma cells, strongly as well as specifically promoted neurite outgrowth (Fig. 4) (ref. 4). The amount of ganglioside used for assay was at a level of a few to twenty micromolar concentrations in the former case, while in the latter only a few nanomolar concentrations were optimal. Most of a similar type of studies so far carried out followed the former. For example, GM3 was found to induce differentiation of human myelogenous leukemia cell line HL-60 into monocyte-mactophages, while neolacto type of gangliosides induced granulocytic differentiation in the same cells. Both ganglioside inducers were added at micromolar concentrations.

Even in the neuritogenic activity of gangliosides, those two contrasted dose dependencies were observed according to the cell type used. Thus, in rat pheochromocytoma PC12, GMl at $50 \mu \mathrm{M}$ did not exhibit any activity in the absence of nerve growth factor (NGF), but strongly enhanced neurite outgrowth and neurofilament expression in coexistence of a low dose of NGF that alone is not sufficient to induce neuronal differentiation. Very recently, it was found that Trk, the high-affinity tyrosine kinase-type receptor for NGF immunoprecipitated together with GM1 in a detergernt-undissociable form and that GM1 binding to the receptor Trk was necessary for full development of the receptor function (ref. 5). The action of GMI was specific and interestingly $\mathrm{N}$-glcosylation of Trk protein seemed to be necessary not only for the association with GM1 but also for its autophosphorylating activity. This study: emphasizes the significance of lipid microdomain surrounding a functional membrane protein or unit, with which a particular type of glycolipids interacts to develop or modulate its function (boundary or annular functional lipids, ref. 6,7).

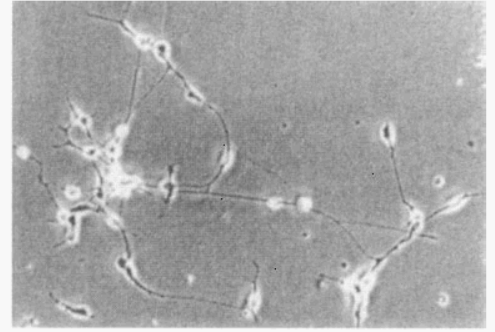

Galb $5 \mathrm{nM}$
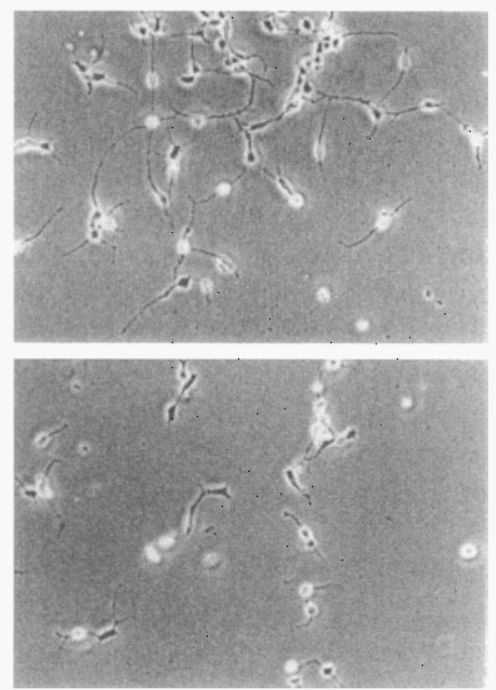

TS-NGF $100 \mathrm{ng} / \mathrm{ml}$

control 


\section{GLYOCORECEPTOR-MEDIATED SIGNALING: ECTO VERSUS ENDO SIGNAL TRANSDUCTION}

In the case of GQ1b-potentiated neuritogenesis, glycoreceptor that specifically recognizes sialooligosaccharide structure of GQ1b plays a central role in the activity. Binding of GQ1b to the receptor activates cell surface-localized ecto protein kinase to phosphorylate a few particular cell surface proteins at serine or threonine site when extracellular ATP is present (ref. 7). Cell membraneimpermeable, small molecular weight inhibitor of protein kinases $\mathrm{K}-252 \mathrm{~b}$ abolished ecto phosphorylation as well as GQ1b-dependent neuritogenesis, providing direct evidence that both phenomena couple to each other (ref. 7). This inhibitor was also found to abolish functional synapse formation of primary cultured rat cerebral cortical neurons, which was measured by monitoring synchronous intracellular $\mathrm{Ca}^{2+}$ oscillation with a video-assisted multi-site fluorometric image analyzer (ref. 8), suggesting that synapse formation is associated with ecto phosphorylation. In the same cell system, of which glycosphingolipids were depleted by D-threo-1-phenyl-2-decanoylamino-3-morpholino-1-propanol (D-PDMP; inhibitor of glucosylceramide synthase as key enzyme for the initiation of glycosphingolipid biosynthesis) (Fig. 1 and 2), synapse formation decreased concomitantly and could be recovered by addition of GQ1b in a dose dependent manner but not by other gangliosides (ref. 9). Thus, several lines of evidence support significance of GQ1b as a neuroactive compound in neuritogenesis as well as synapse formation and that the activity develops via specific carbohydrate recognition of glycoreceptor where initial signaling is carried out by ecto protein kinase-mediated protein phosphorylation with extracellular ATP. This ecto protein kinase signaling system seems to highly develop in neural cells, in particular, neurons. It is to be noted that even GM1-potentiated and NGF-dependent neuritogenesis of PC12 cells was suppressed by D-PDMP, suggesting that ecto signaling pathway may also be in action. It is likely that ecto kinase signaling may be coupled with endo kinase signaling pathway.

Up to now, molecular cloning of more than twelve sialyltransferases have been achieved. When mouse neuroblastoma cells, Neuro 2a, which express mainly GM3 and to a lesser extent other a-series gangliosides, were transfected with cDNA of GD3 synthase ( $\alpha 2,8$-sialyltransferases, SAT-II) (Fig. 2$)$, the cells initiated not only de novo synthesis of b-series gangliosides, GD3 and GQ1b, but also acetylcholine esterase concomitantly (ref. 10). Later it was found that cloned GD3 synthase has also enzymic activity (SAT-V) of the GQlb synthesis from its precursor GT1b (ref. 11) (Fig. 2). Thus, GQlb is physiologically active as well.

\section{NEUROACTIVE SIALYLCHOLESTEROL}

Synthetic $\alpha$ - and $\beta$ - sialylcholesterol both have a potency to promote neurite sprouting in Neuro 2a cells (ref. 12) (Fig. 2). Interestingly it was found that sialylcholesterol, when uptaken into the cells, was quickly transported into cell nuclei, resulting in two-fold increase in transcriptional activity. It is not certain whether the increase is directly associated with neuritogenesis. However, this finding suggests a novel means to manipulate gene expression by carbohydrate compounds. Very recently it was found that both $\alpha$ - and $\beta$-sialylcholesterol promote release of acetylcholine at cholinergic synapse, however, through following two different mechanisms. Namely, $\alpha$-sialylcholesterol accelerated uptake of $\mathrm{Ca}^{2+}$, while $\beta$ epimer enhanced choline uptake through activation of the function of high affinity choline transporter of the synaptic membranes (ref. 13).

\section{GANGLIOSIDE FUNCTION:}

\section{HOUSE KEEPING-OF-LIFE VIEW VERSUS QUALITY-OF-LIFE-VIEW}

In progression of cell biological studies of gangliosides, it became apparent that depending upon the cell type the cells adopt different gangliosides for the same functional purpose and vice versa. For example, GD3 ganglioside is expressed specifically proliferative neurons and astrocytes at activated, proliferative states. Rat embryonic fibroblastic cells 3Y1 expressed GD3 when the cells were transfected and transformed with a oncogenic DNA fragment E1a and the cell growth of these GD3 bearing cells was inhibited reversibly by monoclonal antibody to GD3. However, not all the cells in proliferation express GD3. Moreover, a mouse melanoma cell line GM-95 which is completely deficient in sphingolipids due to the lack of ceramide glucosyltransferase-1 activity (Fig. 2) was reported, the growth of cells being slowed down (ref. 14). As the cells still contained sphingomyelin, they were further treated with neutral sphingomyelinase for complete removal of sphingolipids, resulting in disruption of the cells to substratum adhesion (ref. 15). The results indicate importance of the micro domain of plasma membrane in cell to 
substratum adhesion and also that glycosphingolipids are neither always nor absolutely required for cell growth per se.

At the present, we know neither the reason why the cells behave in such a way regarding the necessity of glycosphingolipids or sphingolipids, nor common structural biological basis to explain such versatile adoption of the glycosphingolipids in responce to demands of cells. Recent success to establish GM2 synthase knockout mice (ref. 16) and galactosylceramide synthase knockout mice (ref. 17) also present a similar enigmatic problem to solve. Both gene-targeted mice were born with seemingly normal ultrastructural appearance except for disorders in spermatogenesis or some electrophysiological parameters such as conduction, although the ganglioside composition of the former mice were solely comprised of GM3 and GD3, and the myelin of the latter mice were entirely deficient in galactosylceramide and sulfatide, both of which have so far been regarded to be essential elements of myelin. Interestingly glucosylceramide which normally does not exist as myelin lipids were now found in myelin, and in progression of age the mice developed abnormality of myelin-related function and regional structural instability of spinal cord (ref. 17). These findings may support important biological roles of gangliosides from the viewpoint of quality-of-life.

\section{References}

1. Nagai, Y. and Iwamori, M. Cellular Biology of Gangliosides, In Biology of the Sialic Acids, (A. Rosenberg, ed.), pp. 197-241. Plenum Press, New York (1995).

2. Ishikawa, D., Taki, T. and Handa, S. Japan. Conf. Biochem. Lipids (J.C.B.L). 39, 106-107 (1997).

3. Bremer, E.G. and Hakomori, S.-I. Biochem, Biophys. Res. Commun. 106, 711-718 (1982).

4. Tsuji, S., Arita, M., Nagai, Y. J. Biochem. 94, 303-306 (1983).

5. Mutoh, T., Tokuda, A., Miyada, T., Hamaguchi, M. and Fujiki, N. Proc. Natl. Acad. Sci., USA.. 92, 5087-5091 (1995).

6. Yamakawa, T. and Nagai, Y. Trends, Biochem. Sci. (TIBS) 3, 128-131 (1978).

7. Nagai, Y. Behavioural Brain Res. 66, 99-104 (1995).

8. Muramoto, K., Kobayashi, K., Nakanishi, S., Matsuda, Y., and Kuroda, Y. Proc. Jpn. Acad. Ser. B. 64, 319-322 (1988).

9. Mizutani, N., Kuroda, Y., Muramoto, K., Kobayashi, K. and Inokuchi, J. Biochem. Biophys. Res. Commun. 22 2, 494-498 (1996).

10. Kojima, N., Kurosawa, N., Nishi, N. and Tsuji, S. J. Biol. Chem. 269, 30451-30456 (1994).

11. Nara, K., Watanabe, Y., Kawashima, I., Tai, T., Nagai, Y. and Sanai, Y. Eur. J. Biochem. 238, 647-652 (1996).

12. Yamashita, T., Tsuji, S. and Nagai, Y. Glycobiology, 1, 149-154 (1991).

13. Tanaka, Y. and Ando, S. Glycoconjugate J. 13. 321-326, (1996); Japan. Conf. Biochem. Lipids (J.C.B.L.), 39, 168-169 (1997).

14. Ichikawa, S., Nakajo, N., Sakiyama, H. and Hirabayashi, Y. Proc. Natl. Acad. Sci., USA.. 9 1, 2703-2707 (1994).

15. Hidari, K. I.-P. J., Ichikawa. S., Fujita, T. Sakiyama, H. and Hirabayashi, Y. J. Biol. Chem. 27 1, 14636-14641 (1996).

16. Takamiya, K., Yamamoto, A. et al., Proc. Natl., Acad., USA. 93, 10662-10667 (1997).

17. Coetzee, T., Fujita, N., Dupree,J., Shi, R., Blight, A., Suzuki, K., Suzuki, K., Popko, B. Cell, 8 6, 209-219 (1996). 